\title{
Self-extinguishing Additive Manufacturing Filament from a Unique Combination of Polylactic Acid and a Polyelectrolyte Complex
}

Thomas J. Kolibaba ${ }^{\dagger}$, Chin-Cheng Shih ${ }^{\ddagger}$, Simone Lazar $^{\dagger}$, Bruce L. Tai ${ }^{\ddagger, *}$, and Jaime C. Grunlan ${ }^{*,+, \neq, \$}$

${ }^{\dagger}$ Department of Chemistry, ${ }^{\ddagger}$ Department of Mechanical Engineering, and ${ }^{\S}$ Department of Materials Science and Engineering, Texas A\&M University, College Station, TX 77843 USA

\section{Experimental Section}

Chemicals and Materials: Hydrochloric acid ( $\mathrm{HCl}$, ACS reagent, 37\%), sodium hydroxide $\left(\mathrm{NaOH}\right.$, ACS reagent, 97\%), and sodium hexametaphosphate (PSP, 65-70\% $\mathrm{P}_{2} \mathrm{O}_{5}$ basis) were purchased from Sigma-Aldrich (St. Louis, MO, USA). Polyvinyl amine (PVA, Lupamin 9095) was acquired from BASF. Polylactic acid (PLA) filament (Natural clear, $1.75 \mathrm{~mm}$ ) was purchased from 3D Solutech (Seattle, WA, USA). All solutions were prepared in deionized (DI) $18 \mathrm{M} \Omega$ water.

Polyelectrolyte Complex Formation: To form the polyelectrolyte complex, two separate, equal volume solutions of $0.25 \mathrm{M}$ PVA and 0.25 M PSP (both with respect to repeat unit molar mass) were adjusted to $\mathrm{pH} 7$, using $5 \mathrm{M} \mathrm{HCl}$ and $5 \mathrm{M} \mathrm{NaOH}$, respectively. These two solutions were then simultaneously mixed under vigorous stirring. The PEC immediately formed and began to precipitate from solution. The mixture stirred for 30 minutes to allow complete formation/precipitation of the complex. After the complex had fully formed, the stirring was stopped to allow for settling of PEC particles. The supernatant from the mixture was decanted, and the PEC was collected and chopped into $\sim 1 \mathrm{~cm}$ pieces with scissors. These pieces were then placed into a jar full of deionized water and rolled for several days, with the water being changed every 8-16 hours, until the conductivity of the supernatant read $<10$ $\mu \mathrm{S} / \mathrm{cm}$ with a handheld conductivity meter (Traceable®, VWR, Radnor, PA, USA), to ensure 
complete removal of all expelled counterions from the complexation process. ${ }^{1}$ The PEC pieces were then dried in a $120{ }^{\circ} \mathrm{C}$ oven overnight, followed by grinding into a fine powder with a coffee grinder.

Filament Extrusion: Chopped pieces (ca. 1-2 cm) of PLA filament were mixed with PEC powder in a 3:1 weight ratio in $4-5 \mathrm{~g}$ batches in scintillation vials. Water was added in a weight equal to that of the PEC powder, which was left to absorb the moisture overnight. The PLA-PEC mixture was then fed into a twin-screw compounder/extruder (HAAKE MiniCTW, Thermo Fisher Scientific, Waltham, MA, USA), set to $185^{\circ} \mathrm{C}$, and mixed at $200 \mathrm{rpm}$ (until a stable torque value $\sim 1 \mathrm{Nm}$ was reached, typically after 15-20 minutes) before being extruded through a $1.7 \mathrm{~mm}$ die in constant-torque mode at $0.07 \mathrm{Nm}$.

Printing: Pieces of filament $\sim 20 \mathrm{~cm}$ long (either pure PLA or PLA-PEC) were fed into a LulzBot Mini 3D printer (Aleph Objects, Inc. Loveland, CO, USA) with a $1.75 \mathrm{~mm}$ hot-end channel. To facilitate quality printing with the PLA-PEC, the printer was slightly modified to have a heater block with a longer melting channel $(\sim 2 \mathrm{~cm})$ and a larger extrusion nozzle $(0.6$ $\mathrm{mm}$ ) to facilitate the slight variation in filament diameter from the extruder utilized. In addition, a nylon tube (with a $2 \mathrm{~mm}$ inner diameter, with a cutout for the feeder to engage and feed filament without buckling) that was attached between the filament entrance and the heat sink to guide filament into the hot-end. PLA and PLA-PEC parts were printed with these modifications at a $200{ }^{\circ} \mathrm{C}$ nozzle temperature and a bed temperature of $50{ }^{\circ} \mathrm{C}$ (both normal for pure PLA) at $3000 \mathrm{~mm} / \mathrm{s}$, with $0.5 \mathrm{~mm}$ filament retraction. 3D models for printing were generated via SolidWorks software (Dassault Systemes, Waltham, MA, USA), while a slicing software (Simplify3D, Cincinnati, OH, USA) was utilized to set up process parameters and plan a printing path.

Characterization: Thermal transitions of the filaments were measured using a differential scanning calorimeter (Q20 DSC, TA Instruments, New Castle, DE, USA) by heating the sample at $10{ }^{\circ} \mathrm{C} / \mathrm{min}$ to $200{ }^{\circ} \mathrm{C}$, then cooling to $0{ }^{\circ} \mathrm{C}$ at $5{ }^{\circ} \mathrm{C} / \mathrm{min}$ to erase thermal history, 
followed by the reported scan from $0-200{ }^{\circ} \mathrm{C}$ at $10{ }^{\circ} \mathrm{C} / \mathrm{min}$ (or $2{ }^{\circ} \mathrm{C} / \mathrm{min}$ in the case of Figure S2). Mechanical properties were measured using a dynamic mechanical analyzer (Q800 DMA, TA Instruments, New Castle, DE, USA) in a single-cantilever arrangement, with a strain rate of $1 \mathrm{~Hz}$ and strain amplitude of $0.1 \%$, while increasing temperature $2{ }^{\circ} \mathrm{C} / \mathrm{min}$ from $30-150{ }^{\circ} \mathrm{C}$. Thermal stability of filaments was determined using a thermogravimetric analyzer (Q50 TGA, TA Instruments, New Castle, DE, USA) under a $60 \mathrm{~mL} / \mathrm{min}$ sample purge flow of air and 40 $\mathrm{mL} / \mathrm{min}$ balance flow of $\mathrm{N}_{2}$. Samples were heated at a rate of $10{ }^{\circ} \mathrm{C} / \mathrm{min}$ to $700{ }^{\circ} \mathrm{C}$, following a 30 minute isothermal hold at $100{ }^{\circ} \mathrm{C}$ to remove any water. Microscale combustion calorimetry experiments were performed by the University of Dayton Research Institute utilizing method A of ASTM D7309 (pyrolysis under nitrogen), heating from $150-600{ }^{\circ} \mathrm{C}$ at $1{ }^{\circ} \mathrm{C} / \mathrm{min}$. Open flame testing was performed with a butane blowtorch (Bernzomatic ST2200, Worthington Industries, Columbus, OH, USA), with its emitter set so that the inner blue flame was $2 \mathrm{~cm}$ in length. Printed parts were suspended from the side by a metal clip and the flame was impinged upon the bottom of the sample from a distance of $3 \mathrm{~cm}$ for $5 \mathrm{~s}$, at which time the flame was removed and the 3D printed part was allowed to burn until either the material self-extinguished or until the flame burned itself out.
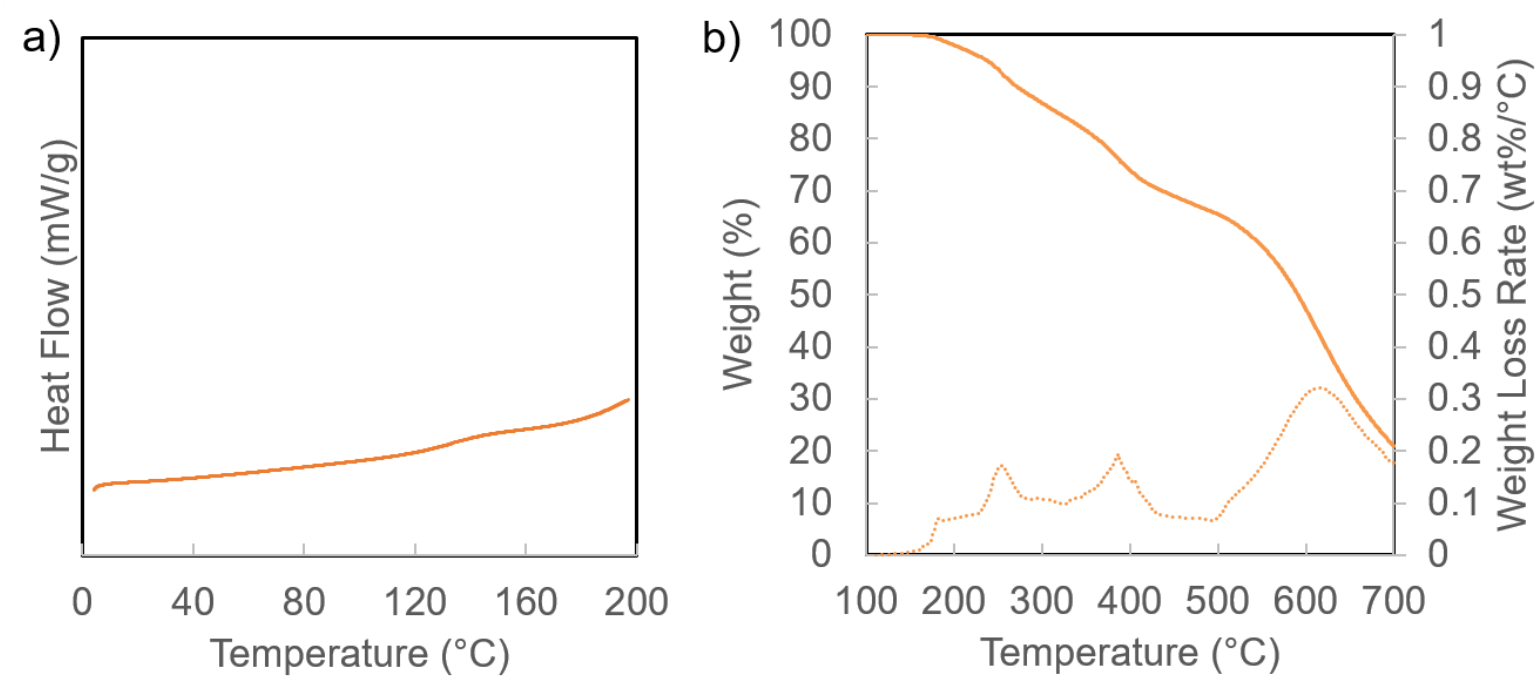
Figure S1. Differential scanning calorimetry (a) and thermogravimetric analysis data showing mass loss (solid line) and mass loss rate (dashed line) for the PVA:PSP polyelectrolyte complex (b).

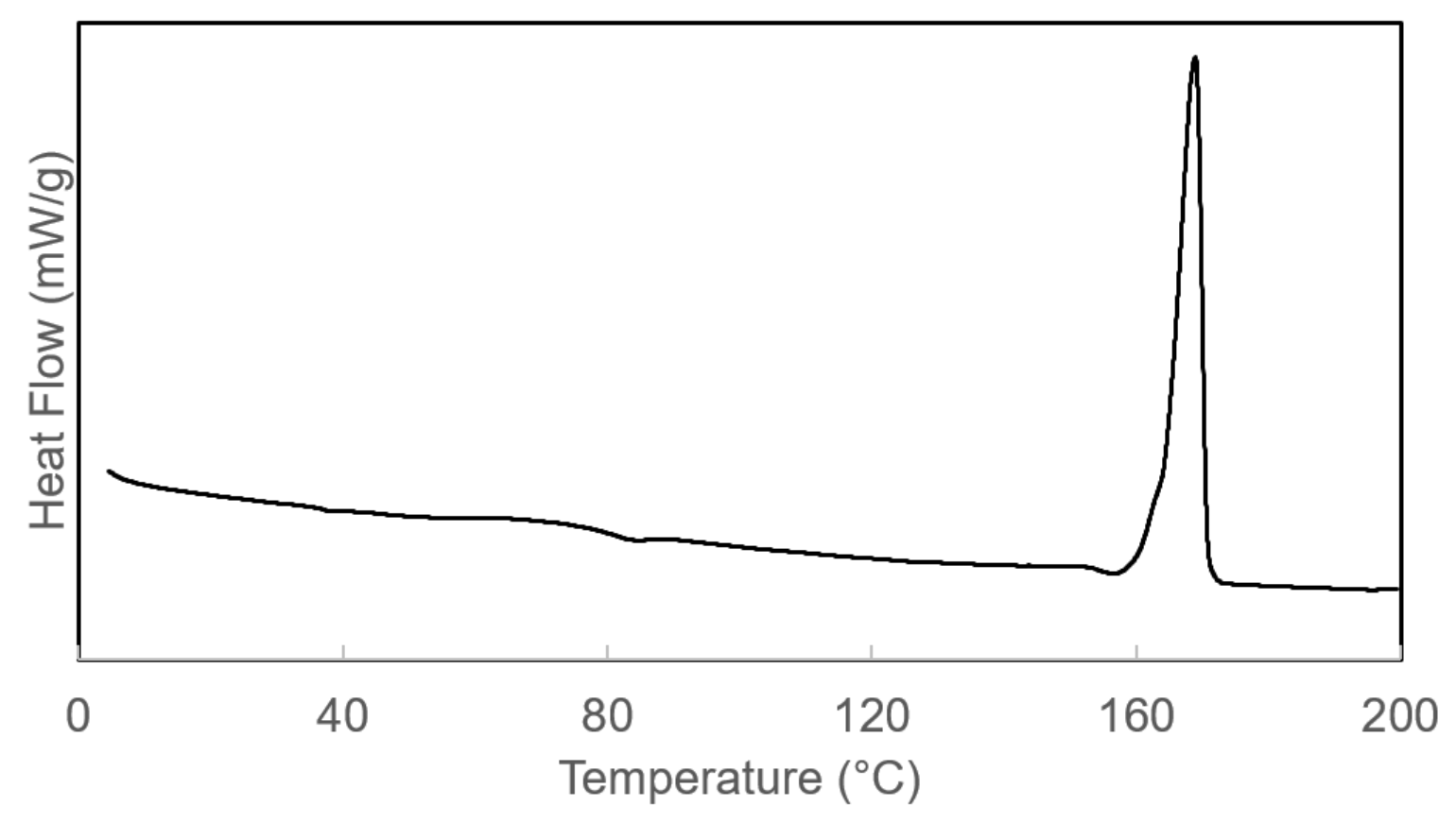

Figure S2. Second-heating DSC trace for PLA-PEC with heating rate of $2 \% \mathrm{~min}$.

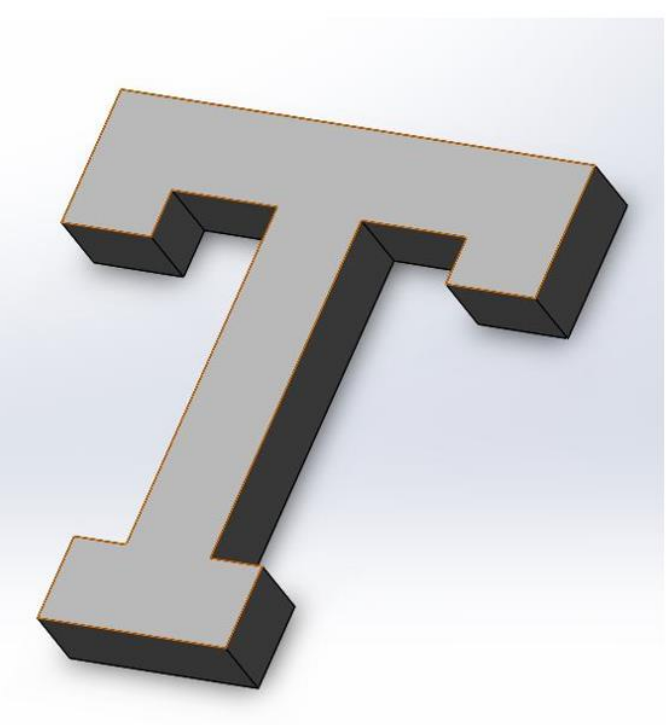

3D Model Generation (SolidWorks)

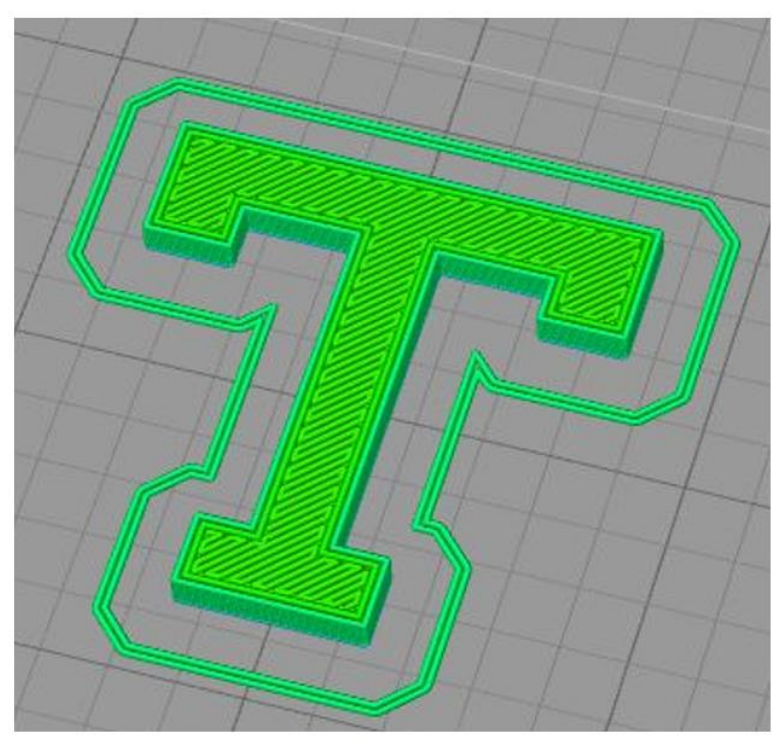

STL Model Slicing (Simplify3D)

Figure S3. 3D model (left) and the sliced printing path (right) used to print parts. 

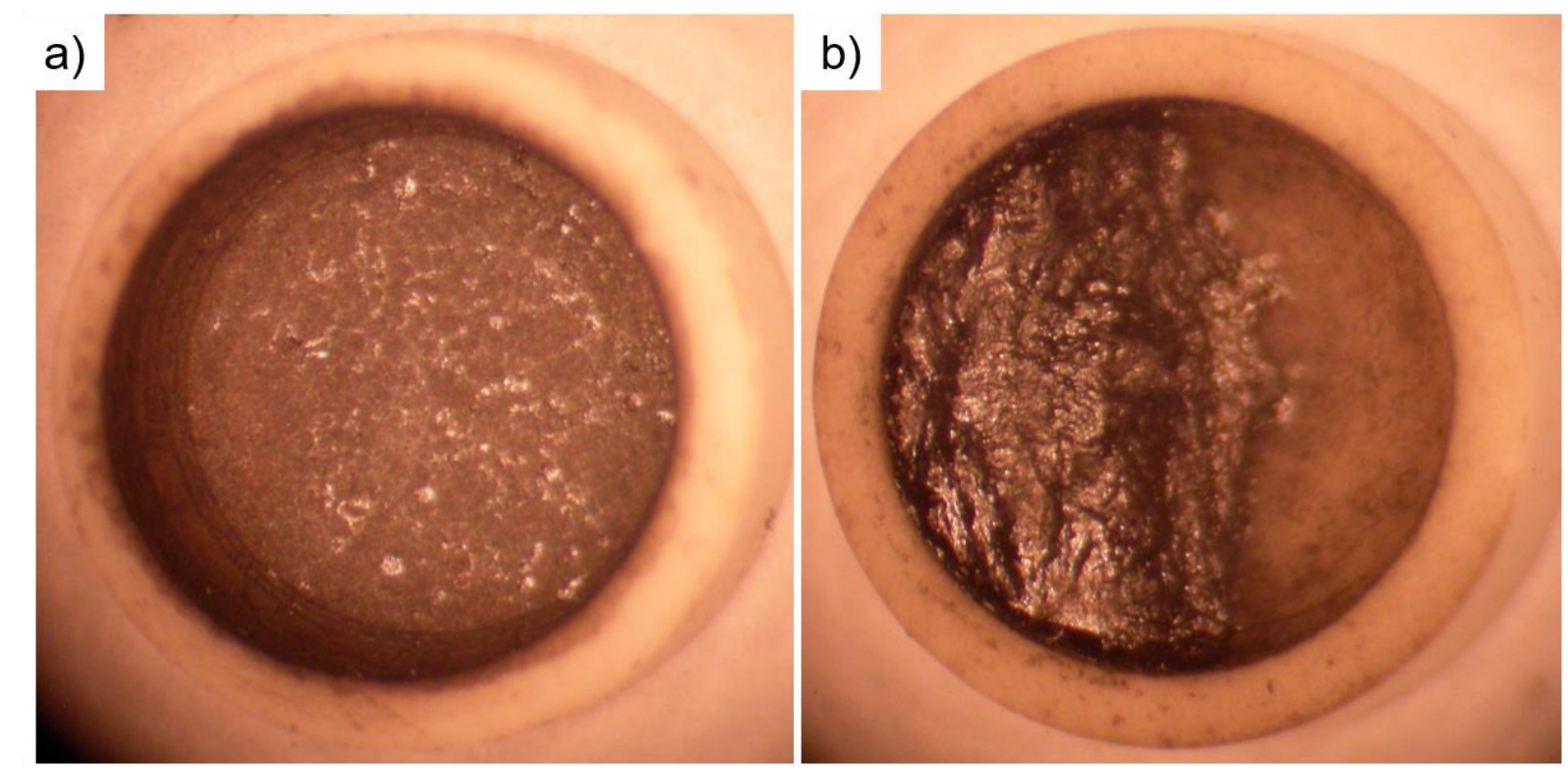

Figure S4. Images of char residue following microscale combustion calorimetry testing of PLA (a) and PLAPEC(b).

\section{References:}

(1) Wang, Q.; Schlenoff, J. B. The Polyelectrolyte Complex/Coacervate Continuum. Macromolecules 2014, 47 (9), 3108-3116. https://doi.org/10.1021/ma500500q. 